\section{Hypertonie: Kardiovaskuläres Risiko steigt früh}

\section{Über den Nutzen einer antihypertensiven Behandlung gibt es generell keinen Zweifel. Es ist allerdings noch relativ wenig untersucht, ob es mit der antihypertensiven Behandlung gelingt, das kardiovaskuläre Risiko wie- der völlig zu normalisieren.}

n die vorliegende Arbeit sind Daten der beiden Studien MESA (Multi-Ethnic Study of Atherosclerosis) und CARDIA

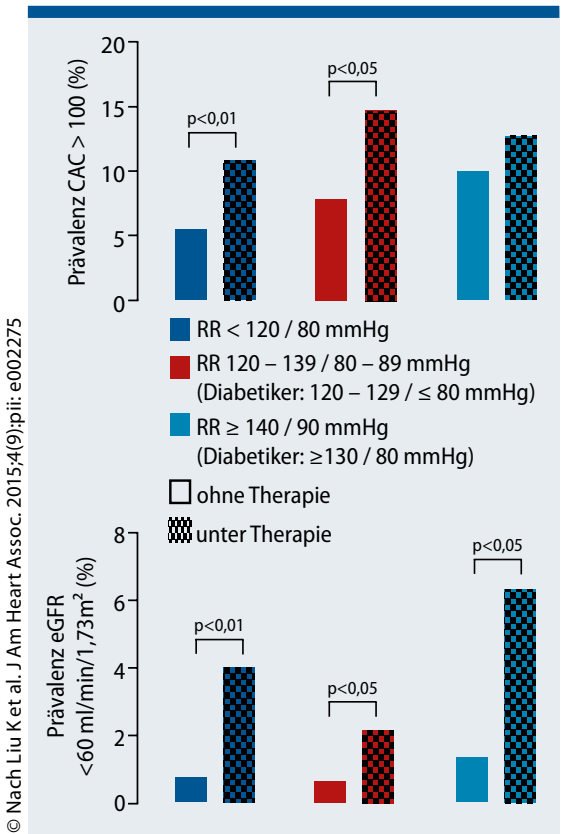

Abb. 1: Oben: Prävalenz eines KoronarkalkScores (CAC) > 100; unten: Prävalenz einer glomerulären Filtrationsrate (eGFR) $<60$ $\mathrm{ml} / \mathrm{min} / 1,73 \mathrm{~m}^{2}$; jeweils mit bzw. ohne antihypertensive Therapie.
(Coronary Artery Risk Development in Young Adults) eingeflossen. In diesen Studien wurde die kardiovaskuläre Prognose von Teilnehmern mit und ohne eine antihypertensive Behandlung verglichen. Die Patienten wurden in drei Blutdruckbereiche eingeordnet: < 120/ $<80 \mathrm{mmHg}, 120$ - $139 \mathrm{mmHg}$ oder $80-$ 89 mmHg (Diabetiker: $120-129 / \leq 80$ $\mathrm{mmHg}$ ) und $\geq 140 \mathrm{mmHg}$ oder $\geq 90$ mmHg (Diabetiker: systolisch $\geq 130$ $\mathrm{mmHg}$ oder diastolisch $\geq 80 \mathrm{mmHg}$ ).

In der MESA-Studie hatten die bei Studienbeginn über 50-jährigen Teilnehmer mit einem Blutdruck $<120 /<80 \mathrm{mmHg}$ unter antihypertensiver Behandlung einen höheren linksventrikulären Massenindex, häufiger eine glomeruläre Filtrationsrate $<60 \mathrm{ml} / \mathrm{min} / 1,73 \mathrm{~m}^{2}$, häufiger einen Koronarkalk-Score $>100$ sowie eine doppelt so hohe Inzidenz kardiovaskulärer Erkrankungen über 9,5 Jahre im Vergleich zu Teilnehmern im gleichen Blutdruckbereich ohne antihypertensive Behandlung (Abb. 1).

In der CARDIA-Studie hatten nach 25 Jahren Teilnehmer mit einem Blutdruck $<120$ / $<80$ mmHg unter antihypertensiver Behandlung zuvor längere Zeit einen höheren Blutdruck sowie ein höheres Risiko von Endorganschäden und subkli- nischer Atherosklerose im Vergleich zu Teilnehmern im selben Blutdruckbereich ohne antihypertensive Behandlung.

Liu K et al. Can Antihypertensive Treatment Restore the Risk of Cardiovascular Disease to Ideal Levels? The Coronary Artery Risk Development in Young Adults (CARDIA) Study and the MultiEthnic Study of Atherosclerosis (MESA). J Am Heart Assoc. 2015; 4(9): pii:e002275

\section{Kommentar}

Diese Ergebnisse lassen nicht etwa Zweifel am Nutzen einer antihypertensiven Behandlung aufkommen. Sie zeigen aber, dass Hypertoniker, auch wenn sie antihypertensiv behandelt werden, sich dadurch nicht wieder völlig auf ein kardiovaskuläres Risiko reduzieren lassen, das bei normotensiven Menschen vorliegt. Die CARDIA-Studie lässt vermuten, dass für diesen Unterschied u. a. auch der Zeitraum eine Rolle spielt, bis das Behandlungsziel erreicht war.

Die Schlussfolgerung für die Praxis lautet daher, dass eine möglichst frühzeitige Behandlung von Hypertonikern hilfreich sein könnte, um das kardiovaskuläre Risiko nach der Blutdruckeinstellung effektiv zu senken. Die Zeit, in der diese Patienten erhöhte Blutdruckwerte haben, bleibt offenbar lange im Gedächtnis ihrer Gefäße haften.

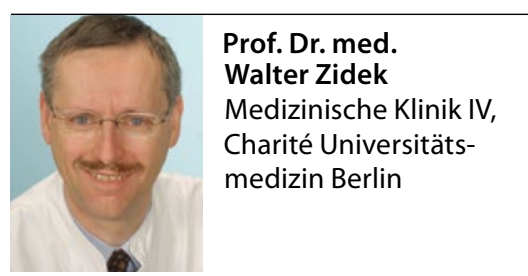

\section{Kardiale Befunde bei 100-Jährigen}

Immer wieder wird darüber spekuliert, dass die alten Alten gesünder sind als die jungen Alten. Doch auch 100-Jährige haben häufig pathologische kardiale Befunde.

n neun spanischen Zentren wurden in zwei Jahren 118 Personen über 100 Jahre rekrutiert, die zuhause (77\%) oder in einem Pflegeheim (23\%) wohnten. Das mittlere Alter der 28 Männer und 90 Frauen betrug 101,5 Jahre (Bereich 100 110 Jahre). Kardiovaskuläre Vorerkrankungen waren häufig (34\% Herzinsuffi- zienz, 15\% zerebrovaskuläre Erkrankungen und $13 \%$ Myokardinfarkt). Begleiterkrankungen waren eher selten und die Probanden fühlten sich überwiegend gesund. Allerdings benötigten nur $11 \%$ keinerlei Hilfe im Alltag, nur $42 \%$ konnten sechs Meter ohne Hilfe gehen. Nach dem Katz-Index waren $42 \%$ nicht bzw. nur gering auf Hilfe angewiesen, bei $21 \%$ bzw. $37 \%$ wurde die Abhängigkeit als mittelschwer oder vollständig eingestuft.

Ein pathologisches EKG hatten $74 \%$ der 100-Jährigen, wobei allerdings auch harmlose Befunde wie Extrasystolen, Schenkelblock, AV-Block 1. Grades und Schrittmacherrhythmus (3\%) mitgezählt wurden. Vorhofflimmern und linksventrikuläre Hypertrophie hatten $21 \%$ bzw. $8 \%$. Pathologische EKG-Befunde waren häufiger bei Rauchern und bei Alkoholkonsum in der Vorgeschichte. Bei der Echokardiografie waren 